Kanishka Perera*, Ratnasingham Shivaji, and Inbo Sim

\title{
A class of semipositone $p$-Laplacian problems with a critical growth reaction term
}

https://doi.org/10.1515/anona-2020-0012

Received December 26, 2017; accepted October 14, 2018.

Abstract: We prove the existence of ground state positive solutions for a class of semipositone $p$-Laplacian problems with a critical growth reaction term. The proofs are established by obtaining crucial uniform $C^{1, \alpha}$ a priori estimates and by concentration compactness arguments. Our results are new even in the semilinear case $p=2$.

Keywords: critical semipositone $p$-Laplacian problems, ground state positive solutions, concentration compactness, uniform $C^{1, \alpha}$ a priori estimates

MSC: Primary 35B33, Secondary 35J92, 35B09, 35B45

\section{Introduction}

Consider the $p$-superlinear semipositone $p$-Laplacian problem

$$
\left\{\begin{aligned}
-\Delta_{p} u & =u^{q-1}-\mu & & \text { in } \Omega \\
u & >0 & & \text { in } \Omega \\
u & =0 & & \text { on } \partial \Omega,
\end{aligned}\right.
$$

where $\Omega$ is a smooth bounded domain in $\mathbb{R}^{N}, 1<p<N, p<q \leq p^{\star}, \mu>0$ is a parameter, and $p^{\star}=N p /(N-p)$ is the critical Sobolev exponent. The scaling $u \mapsto \mu^{1 /(q-1)} u$ transforms the first equation in (1.1) into

$$
-\Delta_{p} u=\mu^{(q-p) /(q-1)}\left(u^{q-1}-1\right),
$$

so in the subcritical case $q<p^{\star}$, it follows from the results in Castro et al.[1] and Chhetri et al.[2] that this problem has a weak positive solution for sufficiently small $\mu>0$ when $p>1$ (see also Unsurangie [3], Allegretto et al.[4], Ambrosetti et al.[5], and Caldwell et al.[6] for the case when $p=2$ ). On the other hand, in the critical case $q=p^{\star}$, it follows from a standard argument involving the Pohozaev identity for the $p$-Laplacian (see Guedda and Véron [7, Theorem 1.1]) that problem (1.1) has no solution for any $\mu>0$ when $\Omega$ is star-shaped. The purpose of the present paper is to show that this situation can be reversed by the addition of lower-order terms, as was observed in the positone case by Brézis and Nirenberg in the celebrated paper [8]. However, this extension to the semipositone case is not straightforward as $u=0$ is no longer a subsolution, making it much harder to find a positive solution as was pointed out in Lions [9]. The positive solutions that we obtain here are ground states, i.e., they minimize the energy among all positive solutions.

*Corresponding Author: Kanishka Perera, Department of Mathematical Sciences, Florida Institute of Technology, Melbourne, FL 32901, USA, E-mail: kperera@fit.edu

Ratnasingham Shivaji, Department of Mathematics and Statistics, University of North Carolina at Greensboro, Greensboro, NC 27412, USA, E-mail: shivaji@uncg.edu

Inbo Sim, Department of Mathematics, University of Ulsan, Ulsan 680-749, Republic of Korea, E-mail: ibsim@ulsan.ac.kr 
We study the Brézis-Nirenberg type critical semipositone $p$-Laplacian problem

$$
\left\{\begin{array}{rlrl}
-\Delta_{p} u & =\lambda u^{p-1}+u^{p^{*}-1}-\mu & & \text { in } \Omega \\
u>0 & & \text { in } \Omega \\
u & =0 & & \text { on } \partial \Omega,
\end{array}\right.
$$

where $\lambda, \mu>0$ are parameters. Let $W_{0}^{1, p}(\Omega)$ be the usual Sobolev space with the norm given by

$$
\|u\|^{p}=\int_{\Omega}|\nabla u|^{p} d x .
$$

For a given $\lambda>0$, the energy of a weak solution $u \in W_{0}^{1, p}(\Omega)$ of problem (1.2) is given by

$$
I_{\mu}(u)=\int_{\Omega}\left(\frac{|\nabla u|^{p}}{p}-\frac{\lambda u^{p}}{p}-\frac{u^{p^{\star}}}{p^{\star}}+\mu u\right) d x
$$

and clearly all weak solutions lie on the set

$$
\mathcal{N}_{\mu}=\left\{u \in W_{0}^{1, p}(\Omega): u>0 \text { in } \Omega \text { and } \int_{\Omega}|\nabla u|^{p} d x=\int_{\Omega}\left(\lambda u^{p}+u^{p^{*}}-\mu u\right) d x\right\} .
$$

We will refer to a weak solution that minimizes $I_{\mu}$ on $\mathcal{N}_{\mu}$ as a ground state. Let

$$
\lambda_{1}=\inf _{u \in W_{0}^{1, p}(\Omega) \backslash\{0\}} \frac{\int_{\Omega}|\nabla u|^{p} d x}{\int_{\Omega}|u|^{p} d x}
$$

be the first Dirichlet eigenvalue of the $p$-Laplacian, which is positive. We will prove the following existence theorem.

Theorem 1.1. If $N \geq p^{2}$ and $\lambda \in\left(0, \lambda_{1}\right)$, then there exists $\mu^{\star}>0$ such that for all $\mu \in\left(0, \mu^{\star}\right)$, problem (1.2) has a ground state solution $u_{\mu} \in C^{1, \alpha}(\bar{\Omega})$ for some $\alpha \in(0,1)$.

The scaling $u \mapsto \mu^{-1 /\left(p^{*}-p\right)} u$ transforms the first equation in the critical semipositone $p$-Laplacian problem

$$
\left\{\begin{aligned}
-\Delta_{p} u & =\lambda u^{p-1}+\mu\left(u^{p^{*}-1}-1\right) & & \text { in } \Omega \\
u & >0 & & \text { in } \Omega \\
u & =0 & & \text { on } \partial \Omega
\end{aligned}\right.
$$

into

$$
-\Delta_{p} u=\lambda u^{p-1}+u^{p^{*}-1}-\mu^{\left(p^{*}-1\right) /\left(p^{*}-p\right)},
$$

so as an immediate corollary we have the following existence theorem for problem (1.4).

Theorem 1.2. If $N \geq p^{2}$ and $\lambda \in\left(0, \lambda_{1}\right)$, then there exists $\mu^{\star}>0$ such that for all $\mu \in\left(0, \mu^{\star}\right)$, problem (1.4) has a ground state solution $u_{\mu} \in C^{1, \alpha}(\bar{\Omega})$ for some $\alpha \in(0,1)$.

We would like to emphasize that Theorems 1.1 and 1.2 are new even in the semilinear case $p=2$.

The outline of the proof of Theorem 1.1 is as follows. We consider the modified problem

$$
\left\{\begin{aligned}
-\Delta_{p} u & =\lambda u_{+}^{p-1}+u_{+}^{p^{*}-1}-\mu f(u) & & \text { in } \Omega \\
u & =0 & & \text { on } \partial \Omega,
\end{aligned}\right.
$$


where $u_{+}(x)=\max \{u(x), 0\}$ and

$$
f(t)= \begin{cases}1, & t \geq 0 \\ 1-|t|^{p-1}, & -1<t<0 \\ 0, & t \leq-1 .\end{cases}
$$

Weak solutions of this problem coincide with critical points of the $C^{1}$-functional

$$
\begin{aligned}
I_{\mu}(u)=\int_{\Omega}\left(\frac{|\nabla u|^{p}}{p}-\frac{\lambda u_{+}^{p}}{p}-\frac{u_{+}^{p^{*}}}{p^{\star}}\right) & d x+\mu\left[\int_{\{u \geq 0\}} u d x\right. \\
& \left.+\int_{\{-1<u<0\}}\left(u-\frac{|u|^{p-1} u}{p}\right) d x-\left(1-\frac{1}{p}\right)|\{u \leq-1\}|\right], \quad u \in W_{0}^{1, p}(\Omega),
\end{aligned}
$$

where $|\cdot|$ denotes the Lebesgue measure in $\mathbb{R}^{N}$. Recall that $I_{\mu}$ satisfies the Palais-Smale compactness condition at the level $c \in \mathbb{R}$, or the (PS) $)_{c}$ condition for short, if every sequence $\left(u_{j}\right) \subset W_{0}^{1, p}(\Omega)$ such that $I_{\mu}\left(u_{j}\right) \rightarrow c$ and $I_{\mu}^{\prime}\left(u_{j}\right) \rightarrow 0$, called a (PS) $)_{c}$ sequence for $I_{\mu}$, has a convergent subsequence. As we will see in Lemma 2.1 in the next section, it follows from concentration compactness arguments that $I_{\mu}$ satisfies the (PS) ${ }_{c}$ condition for all

$$
c<\frac{1}{N} S^{N / p}-\left(1-\frac{1}{p}\right) \mu|\Omega|,
$$

where $S$ is the best Sobolev constant (see (2.1)). First we will construct a mountain pass level below this threshold for compactness for all sufficiently small $\mu>0$. This part of the proof is more or less standard. The novelty of the paper lies in the fact that the solution $u_{\mu}$ of the modified problem (1.5) thus obtained is positive, and hence also a solution of our original problem (1.2), if $\mu$ is further restricted. Note that this does not follow from the strong maximum principle as usual since $-\mu f(0)<0$. This is precisely the main difficulty in finding positive solutions of semipositone problems (see Lions [9]). We will prove that for every sequence $\mu_{j} \rightarrow 0$, a subsequence of $u_{\mu_{j}}$ is positive in $\Omega$. The idea is to show that a subsequence of $u_{\mu_{j}}$ converges in $C_{0}^{1}(\bar{\Omega})$ to a solution of the limit problem

$$
\left\{\begin{aligned}
-\Delta_{p} u & =\lambda u^{p-1}+u^{p^{*}-1} & & \text { in } \Omega \\
u & >0 & & \text { in } \Omega \\
u & =0 & & \text { on } \partial \Omega .
\end{aligned}\right.
$$

This requires a uniform $C^{1, \alpha}(\bar{\Omega})$ estimate of $u_{\mu_{j}}$ for some $\alpha \in(0,1)$. We will obtain such an estimate by showing that $u_{\mu_{j}}$ is uniformly bounded in $W_{0}^{1, p}(\Omega)$ and uniformly equi-integrable in $L^{p^{*}}(\Omega)$, and applying a result of de Figueiredo et al.[10]. The proof of uniform equi-integrability in $L^{p^{*}}(\Omega)$ involves a second (nonstandard) application of the concentration compactness principle. Finally, we use the mountain pass characterization of our solution to show that it is indeed a ground state.

Remark 1.3. Establishing the existence of solutions to the critical semipositone problem

$$
\left\{\begin{aligned}
-\Delta_{p} u & =\mu\left(u^{p-1}+u^{p^{*}-1}-1\right) & & \text { in } \Omega \\
u & >0 & & \text { in } \Omega \\
u & =0 & & \text { on } \partial \Omega
\end{aligned}\right.
$$

for small $\mu$ remains open. 


\section{Preliminaries}

Let

$$
S=\inf _{u \in W_{0}^{1, p}(\Omega) \backslash\{0\}} \frac{\int_{\Omega}|\nabla u|^{p} d x}{\left(\int_{\Omega}|u|^{p^{*}} d x\right)^{p / p^{*}}}
$$

be the best constant in the Sobolev inequality, which is independent of $\Omega$. The proof of Theorem 1.1 will make use of the following compactness result.

Lemma 2.1. For any fixed $\lambda, \mu>0, I_{\mu}$ satisfies the $(\mathrm{PS})_{c}$ condition for all

$$
c<\frac{1}{N} S^{N / p}-\left(1-\frac{1}{p}\right) \mu|\Omega| .
$$

Proof. Let $\left(u_{j}\right)$ be a $(\mathrm{PS})_{c}$ sequence. First we show that $\left(u_{j}\right)$ is bounded. We have

$$
\begin{aligned}
I_{\mu}\left(u_{j}\right)=\int_{\Omega}\left(\frac{\left|\nabla u_{j}\right|^{p}}{p}-\frac{\lambda u_{j+}^{p}}{p}-\frac{u_{j+}^{p^{*}}}{p^{\star}}\right) d x+\mu & {\left[\int_{\left\{u_{j} \geq 0\right\}} u_{j} d x\right.} \\
& \left.+\int_{\left\{-1<u_{j}<0\right\}}\left(u_{j}-\frac{\left|u_{j}\right|^{p-1} u_{j}}{p}\right) d x-\left(1-\frac{1}{p}\right)\left|\left\{u_{j} \leq-1\right\}\right|\right]=c+o(1)
\end{aligned}
$$

and

$$
\begin{aligned}
I_{\mu}^{\prime}\left(u_{j}\right) v=\int_{\Omega}\left(\left|\nabla u_{j}\right|^{p-2} \nabla u_{j} \cdot \nabla v-\lambda u_{j+}^{p-1} v-u_{j+}^{p^{*}-1} v\right) d x+\mu\left[\int_{\left\{u_{j} \geq 0\right\}} v d x\right. \\
\left.+\int_{\left\{-1<u_{j}<0\right\}}\left(1-\left|u_{j}\right|^{p-1}\right) v d x\right]=\mathrm{o}(1)\|v\| \quad \forall v \in W_{0}^{1, p}(\Omega) .
\end{aligned}
$$

Taking $v=u_{j}$ in (2.4), dividing by $p$, and subtracting from (2.3) gives

$$
\frac{1}{N} \int_{\Omega} u_{j_{+}}^{p^{*}} d x \leq c+\left(1-\frac{1}{p}\right) \mu|\Omega|+\mathrm{o}(1)\left(\left\|u_{j}\right\|+1\right),
$$

and it follows from this, (2.3), and the Hölder inequality that $\left(u_{j}\right)$ is bounded in $W_{0}^{1, p}(\Omega)$.

Since $\left(u_{j}\right)$ is bounded, so is $\left(u_{j_{+}}\right)$, a renamed subsequence of which then converges to some $v \geq 0$ weakly in $W_{0}^{1, p}(\Omega)$, strongly in $L^{q}(\Omega)$ for all $q \in\left[1, p^{\star}\right)$ and a.e.in $\Omega$, and

$$
\left|\nabla u_{j+}\right|^{p} d x \stackrel{w^{*}}{\longrightarrow} \kappa, \quad u_{j+}^{p^{*}} d x \stackrel{w^{*}}{\longrightarrow} v
$$

in the sense of measures, where $\kappa$ and $v$ are bounded nonnegative measures on $\bar{\Omega}$ (see, e.g., Folland [11]). By the concentration compactness principle of Lions $[12,13]$, then there exist an at most countable index set $I$ and points $x_{i} \in \bar{\Omega}, i \in I$ such that

$$
\kappa \geq|\nabla v|^{p} d x+\sum_{i \in I} \kappa_{i} \delta_{x_{i}}, \quad v=v^{p^{\star}} d x+\sum_{i \in I} v_{i} \delta_{x_{i}}
$$


where $\kappa_{i}, v_{i}>0$ and $v_{i}^{p / p^{*}} \leq \kappa_{i} / S$. We claim that $I=\emptyset$. Suppose by contradiction that there exists $i \in I$. Let $\varphi: \mathbb{R}^{N} \rightarrow[0,1]$ be a smooth function such that $\varphi(x)=1$ for $|x| \leq 1$ and $\varphi(x)=0$ for $|x| \geq 2$. Then set

$$
\varphi_{i, \rho}(x)=\varphi\left(\frac{x-x_{i}}{\rho}\right), \quad x \in \mathbb{R}^{N}
$$

for $i \in I$ and $\rho>0$, and note that $\varphi_{i, \rho}: \mathbb{R}^{N} \rightarrow[0,1]$ is a smooth function such that $\varphi_{i, \rho}(x)=1$ for $\left|x-x_{i}\right| \leq \rho$ and $\varphi_{i, \rho}(x)=0$ for $\left|x-x_{i}\right| \geq 2 \rho$. The sequence $\left(\varphi_{i, \rho} u_{j+}\right)$ is bounded in $W_{0}^{1, p}(\Omega)$ and hence taking $v=\varphi_{i, \rho} u_{j+}$ in (2.4) gives

$$
\int_{\Omega}\left(\varphi_{i, \rho}\left|\nabla u_{j+}\right|^{p}+u_{j+}\left|\nabla u_{j+}\right|^{p-2} \nabla u_{j+} \cdot \nabla \varphi_{i, \rho}-\lambda \varphi_{i, \rho} u_{j+}^{p}-\varphi_{i, \rho} u_{j+}^{p^{*}}+\mu \varphi_{i, \rho} u_{j+}\right) d x=\mathrm{o}(1) .
$$

By (2.6),

$$
\int_{\Omega} \varphi_{i, \rho}\left|\nabla u_{j+}\right|^{p} d x \rightarrow \int_{\Omega} \varphi_{i, \rho} d \kappa, \quad \int_{\Omega} \varphi_{i, \rho} u_{j+}^{p^{*}} d x \rightarrow \int_{\Omega} \varphi_{i, \rho} d v .
$$

Denoting by $C$ a generic positive constant independent of $j$ and $\rho$,

$$
\left|\int_{\Omega}\left(u_{j_{+}}\left|\nabla u_{j_{+}}\right|^{p-2} \nabla u_{j_{+}} \cdot \nabla \varphi_{i, \rho}-\lambda \varphi_{i, \rho} u_{j+}^{p}+\mu \varphi_{i, \rho} u_{j_{+}}\right) d x\right| \leq C\left[\left(\frac{1}{\rho}+\mu\right) I_{j}^{1 / p}+I_{j}\right],
$$

where

$$
I_{j}:=\int_{\Omega \cap B_{2 \rho}\left(x_{i}\right)} u_{j+}^{p} d x \rightarrow \int_{\Omega \cap B_{2 \rho}\left(x_{i}\right)} v^{p} d x \leq C \rho^{p}\left(\int_{\Omega \cap B_{2 \rho}\left(x_{i}\right)} v^{p^{*}} d x\right)^{p / p^{*}} .
$$

So passing to the limit in (2.8) gives

$$
\int_{\Omega} \varphi_{i, \rho} d \kappa-\int_{\Omega} \varphi_{i, \rho} d v \leq C\left[(1+\mu \rho)\left(\int_{\Omega \cap B_{2 \rho}\left(x_{i}\right)} v^{p^{*}} d x\right)^{1 / p^{*}}+\int_{\Omega \cap B_{2 \rho}\left(x_{i}\right)} v^{p} d x\right] .
$$

Letting $\rho \vee 0$ and using (2.7) now gives $\kappa_{i} \leq v_{i}$, which together with $v_{i}>0$ and $v_{i}^{p / p^{*}} \leq \kappa_{i} / S$ then gives $v_{i} \geq S^{N / p}$. On the other hand, passing to the limit in (2.5) and using (2.6) and (2.7) gives

$$
v_{i} \leq N\left[c+\left(1-\frac{1}{p}\right) \mu|\Omega|\right]<S^{N / p}
$$

by (2.2), a contradiction. Hence $I=\emptyset$ and

$$
\int_{\Omega} u_{j+}^{p^{*}} d x \rightarrow \int_{\Omega} v^{p^{*}} d x
$$

Passing to a further subsequence, $u_{j}$ converges to some $u$ weakly in $W_{0}^{1, p}(\Omega)$, strongly in $L^{q}(\Omega)$ for all $q \in\left[1, p^{\star}\right)$, and a.e.in $\Omega$. Since

$$
\left|u_{j+}^{p^{*}-1}\left(u_{j}-u\right)\right| \leq u_{j+}^{p^{*}}+u_{j+}^{p^{*}-1}|u| \leq\left(2-\frac{1}{p^{*}}\right) u_{j+}^{p^{*}}+\frac{1}{p^{*}}|u|^{p^{*}}
$$

by Young’s inequality,

$$
\int_{\Omega} u_{j+}^{p^{*}-1}\left(u_{j}-u\right) d x \rightarrow 0
$$

by (2.9) and the dominated convergence theorem. Then taking $v=u_{j}-u$ in (2.4) gives

$$
\int_{\Omega}\left|\nabla u_{j}\right|^{p-2} \nabla u_{j} \cdot \nabla\left(u_{j}-u\right) d x \rightarrow 0,
$$

so $u_{j} \rightarrow u$ in $W_{0}^{1, p}(\Omega)$ for a renamed subsequence (see, e.g., Perera et al.[14, Proposition 1.3]). 
The infimum in (2.1) is attained by the family of functions

$$
u_{\varepsilon}(x)=\frac{C_{N, p} \varepsilon^{(N-p) / p^{2}}}{\left(\varepsilon+|x|^{p /(p-1)}\right)^{(N-p) / p}}, \quad \varepsilon>0
$$

when $\Omega=\mathbb{R}^{N}$, where the constant $C_{N, p}>0$ is chosen so that

$$
\int_{\mathbb{R}^{N}}\left|\nabla u_{\varepsilon}\right|^{p} d x=\int_{\mathbb{R}^{N}} u_{\varepsilon}^{p^{*}} d x=S^{N / p} .
$$

Without loss of generality, we may assume that $0 \in \Omega$. Let $r>0$ be so small that $B_{2 r}(0) \subset \Omega$, take a function $\psi \in C_{0}^{\infty}\left(B_{2 r}(0),[0,1]\right)$ such that $\psi=1$ on $B_{r}(0)$, and set

$$
\tilde{u}_{\varepsilon}(x)=\psi(x) u_{\varepsilon}(x), \quad v_{\varepsilon}(x)=\frac{\tilde{u}_{\varepsilon}(x)}{\left(\int_{\Omega} \tilde{u}_{\varepsilon}^{p^{*}} d x\right)^{1 / p^{*}}},
$$

so that $\int_{\Omega} v_{\varepsilon}^{p^{*}} d x=1$. Then we have the well-known estimates

$$
\begin{gathered}
\int_{\Omega}\left|\nabla v_{\varepsilon}\right|^{p} d x \leq S+C \varepsilon^{(N-p) / p}, \\
\int_{\Omega} v_{\varepsilon}^{p} d x \geq \begin{cases}\frac{1}{C} \varepsilon^{p-1}, & N>p^{2} \\
\frac{1}{C} \varepsilon^{p-1}|\log \varepsilon|, & N=p^{2},\end{cases}
\end{gathered}
$$

where $C=C(N, p)>0$ is a constant (see, e.g., Drábek and Huang [15]).

\section{Proof of Theorem 1.1}

First we show that $I_{\mu}$ has a uniformly positive mountain pass level below the threshold for compactness given in Lemma 2.1 for all sufficiently small $\mu>0$. Let $v_{\varepsilon}$ be as in the last section.

Lemma 3.1. There exist $\mu_{0}, \rho, c_{0}>0, R>\rho$, and $\beta<\frac{1}{N} S^{N / p}$ such that the following hold for all $\mu \in\left(0, \mu_{0}\right)$ :

(i) $\|u\|=\rho \Rightarrow I_{\mu}(u) \geq c_{0}$,

(ii) $\quad I_{\mu}\left(t v_{\varepsilon}\right) \leq 0$ for all $t \geq R$ and $\varepsilon \in(0,1]$,

(iii) denoting by $\Gamma=\left\{y \in C\left([0,1], W_{0}^{1, p}(\Omega)\right): y(0)=0, y(1)=R v_{\varepsilon}\right\}$ the class of paths joining the origin to $R v_{\varepsilon}$,

$$
c_{0} \leq c_{\mu}:=\inf _{y \in \Gamma} \max _{u \in y([0,1])} I_{\mu}(u) \leq \beta-\left(1-\frac{1}{p}\right) \mu|\Omega|
$$

for all sufficiently small $\varepsilon>0$,

(iv) $\quad I_{\mu}$ has a critical point $u_{\mu}$ at the level $c_{\mu}$.

Proof. By (1.3) and (2.1),

$$
I_{\mu}(u) \geq \frac{1}{p}\left(1-\frac{\lambda}{\lambda_{1}}\right)\|u\|^{p}-\frac{S^{-p^{*} / p}}{p^{\star}}\|u\|^{p^{*}}-\left(1-\frac{1}{p}\right) \mu|\Omega|,
$$

and (i) follows from this for sufficiently small $\rho, c_{0}, \mu>0$ since $\lambda<\lambda_{1}$. 
Since $v_{\varepsilon} \geq 0$,

$$
I_{\mu}\left(t v_{\varepsilon}\right)=\frac{t^{p}}{p} \int_{\Omega}\left(\left|\nabla v_{\varepsilon}\right|^{p}-\lambda v_{\varepsilon}^{p}\right) d x-\frac{t^{p^{\star}}}{p^{\star}}+\mu t \int_{\Omega} v_{\varepsilon} d x
$$

for $t \geq 0$. By the Hölder's and Young's inequalities,

$$
\mu t \int_{\Omega} v_{\varepsilon} d x \leq \mu t|\Omega|^{1-1 / p}\left(\int_{\Omega} v_{\varepsilon}^{p} d x\right)^{1 / p} \leq C_{\lambda} \mu^{p /(p-1)}+\frac{\lambda t^{p}}{2 p} \int_{\Omega} v_{\varepsilon}^{p} d x,
$$

where

so

$$
C_{\lambda}=\left(1-\frac{1}{p}\right)\left(\frac{2}{\lambda}\right)^{1 /(p-1)}|\Omega|
$$

$$
I_{\mu}\left(t v_{\varepsilon}\right) \leq \frac{t^{p}}{p} \int_{\Omega}\left(\left|\nabla v_{\varepsilon}\right|^{p}-\frac{\lambda}{2} v_{\varepsilon}^{p}\right) d x-\frac{t^{p^{\star}}}{p^{\star}}+C_{\lambda} \mu^{p /(p-1)} .
$$

Then by (2.10) and for $\varepsilon, \mu \in(0,1]$,

$$
I_{\mu}\left(t v_{\varepsilon}\right) \leq(S+C) \frac{t^{p}}{p}-\frac{t^{p^{\star}}}{p^{\star}}+C_{\lambda},
$$

from which (ii) follows for sufficiently large $R>\rho$.

The first inequality in (3.1) is immediate from (i) since $R>\rho$. Maximizing the right-hand side of (3.2) over $t \geq 0$ gives

$$
c_{\mu} \leq \frac{1}{N}\left[\int_{\Omega}\left(\left|\nabla v_{\varepsilon}\right|^{p}-\frac{\lambda}{2} v_{\varepsilon}^{p}\right) d x\right]^{N / p}+C_{\lambda} \mu^{p /(p-1)},
$$

and (2.10) and (2.11) imply that the integral on the right-hand side is strictly less than $S$ for all sufficiently small $\varepsilon>0$ since $N \geq p^{2}$ and $\lambda>0$, so the second inequality in (3.1) holds for sufficiently small $\mu>0$.

Finally, (iv) follows from (i)-(iii), Lemma 2.1, and the mountain pass lemma (see Ambrosetti and Rabinowitz [16]).

Next we show that $u_{\mu}$ is uniformly bounded in $W_{0}^{1, p}(\Omega)$ and uniformly equi-integrable in $L^{p^{*}}(\Omega)$, and hence also uniformly bounded in $C^{1, \alpha}(\bar{\Omega})$ for some $\alpha \in(0,1)$ by de Figueiredo et al. [10, Proposition 3.7], for all sufficiently small $\mu \in\left(0, \mu_{0}\right)$.

Lemma 3.2. There exists $\mu_{\star} \in\left(0, \mu_{0}\right]$ such that the following hold for all $\mu \in\left(0, \mu_{\star}\right)$ :

(i) $\quad u_{\mu}$ is uniformly bounded in $W_{0}^{1, p}(\Omega)$,

(iii) $\quad u_{\mu}$ is uniformly bounded in $C^{1, \alpha}(\bar{\Omega})$ for some $\alpha \in(0,1)$.

Proof. We have

$$
\begin{aligned}
I_{\mu}\left(u_{\mu}\right)=\int_{\Omega}\left(\frac{\left|\nabla u_{\mu}\right|^{p}}{p}-\frac{\lambda u_{\mu+}^{p}}{p}-\frac{u_{\mu+}^{p^{*}}}{p^{\star}}\right) d x+\mu & {\left[\int_{\left\{u_{\mu} \geq 0\right\}} u_{\mu} d x\right.} \\
& \left.+\int_{\left\{-1<u_{\mu}<0\right\}}\left(u_{\mu}-\frac{\left|u_{\mu}\right|^{p-1} u_{\mu}}{p}\right) d x-\left(1-\frac{1}{p}\right)\left|\left\{u_{\mu} \leq-1\right\}\right|\right]=c_{\mu}
\end{aligned}
$$


and

$$
\begin{aligned}
I_{\mu}^{\prime}\left(u_{\mu}\right) v=\int_{\Omega}\left(\left|\nabla u_{\mu}\right|^{p-2} \nabla u_{\mu} \cdot \nabla v-\lambda u_{\mu+}^{p-1} v-u_{\mu+}^{p^{*}-1} v\right) d x+\mu & {\left[\int_{\left\{u_{\mu} \geq 0\right\}} v d x\right.} \\
& \left.+\int_{\left\{-1<u_{\mu}<0\right\}}\left(1-\left|u_{\mu}\right|^{p-1}\right) v d x\right]=0 \quad \forall v \in W_{0}^{1, p}(\Omega) .
\end{aligned}
$$

Taking $v=u_{\mu}$ in (3.4), dividing by $p$, and subtracting from (3.3) gives

$$
\frac{1}{N} \int_{\Omega} u_{\mu+}^{p^{*}} d x \leq c_{\mu}+\left(1-\frac{1}{p}\right) \mu|\Omega| \leq \beta
$$

by (3.1), and (i) follows from this, (3.4) with $v=u_{\mu}$, and the Hölder inequality.

If (ii) does not hold, then there exist sequences $\mu_{j} \rightarrow 0$ and $\left(E_{j}\right)$ with $\left|E_{j}\right| \rightarrow 0$ such that

$$
\underline{\lim } \int_{E_{j}}\left|u_{\mu_{j}}\right|^{p^{*}} d x>0 .
$$

Since $\left(u_{\mu_{j}}\right)$ is bounded by $(i)$, so is $\left(u_{\mu_{j^{+}}}\right)$, a renamed subsequence of which then converges to some $v \geq 0$ weakly in $W_{0}^{1, p}(\Omega)$, strongly in $L^{q}(\Omega)$ for all $q \in\left[1, p^{\star}\right)$ and a.e.in $\Omega$, and

$$
\left|\nabla u_{\mu_{j^{+}}}\right|^{p} d x \stackrel{w^{\star}}{\longrightarrow} \kappa, \quad u_{\mu_{j^{+}}}^{p^{\star}} d x \stackrel{w^{\star}}{\longrightarrow} v
$$

in the sense of measures, where $\kappa$ and $v$ are bounded nonnegative measures on $\bar{\Omega}$. By Lions [12,13], then there exist an at most countable index set $I$ and points $x_{i} \in \bar{\Omega}, i \in I$ such that

$$
\kappa \geq|\nabla v|^{p} d x+\sum_{i \in I} \kappa_{i} \delta_{x_{i}}, \quad v=v^{p^{*}} d x+\sum_{i \in I} v_{i} \delta_{x_{i}},
$$

where $\kappa_{i}, v_{i}>0$ and $v_{i}^{p / p^{*}} \leq \kappa_{i} / S$. Suppose $I$ is nonempty, say, $i \in I$. An argument similar to that in the proof of Lemma 2.1 shows that $\kappa_{i} \leq v_{i}$, so $v_{i} \geq S^{N / p}$. On the other hand, passing to the limit in (3.5) with $\mu=\mu_{j}$ and using (3.7) and (3.8) gives $v_{i} \leq N \beta<S^{N / p}$, a contradiction. Hence $I=\emptyset$ and

$$
\int_{\Omega} u_{\mu_{j}+}^{p^{*}} d x \rightarrow \int_{\Omega} v^{p^{*}} d x .
$$

As in the proof of Lemma 2.1, a further subsequence of $\left(u_{\mu_{j}}\right)$ then converges to some $u$ in $W_{0}^{1, p}(\Omega)$, and hence also in $L^{p^{*}}(\Omega)$, and a.e.in $\Omega$. Then

$$
\int_{E_{j}}\left|u_{\mu_{j}}\right|^{p^{*}} d x \leq\left.\int_{\Omega}|| u_{\mu_{j}}\right|^{p^{*}}-\left.|u|^{p^{*}}\left|d x+\int_{E_{j}}\right| u\right|^{p^{*}} d x \rightarrow 0,
$$

contradicting (3.6).

Finally, (iii) follows from (i), (ii), and de Figueiredo et al.[10, Proposition 3.7].

We are now ready to prove Theorem 1.1.

Proof of Theorem 1.1. We claim that $u_{\mu}$ is positive in $\Omega$, and hence a weak solution of problem (1.2), for all sufficiently small $\mu \in\left(0, \mu_{\star}\right)$. It suffices to show that for every sequence $\mu_{j} \rightarrow 0$, a subsequence of $u_{\mu_{j}}$ is 
positive in $\Omega$. By Lemma 3.2 (iii), a renamed subsequence of $u_{\mu_{j}}$ converges to some $u$ in $C_{0}^{1}(\bar{\Omega})$. We have

$$
\begin{aligned}
I_{\mu_{j}}\left(u_{\mu_{j}}\right)=\int_{\Omega}\left(\frac{\left|\nabla u_{\mu_{j}}\right|^{p}}{p}-\frac{\lambda u_{\mu_{j^{+}}}^{p}}{p}-\frac{u_{\mu_{j^{+}}}^{p^{*}}}{p^{*}}\right) d x+\mu_{j}\left[\int_{\left\{u_{\mu_{j}} \geq 0\right\}} u_{\mu_{j}} d x\right. \\
\left.+\int_{\left\{-1<u_{\left.\mu_{j}<0\right\}}\right.}\left(u_{\mu_{j}}-\frac{\left|u_{\mu_{j}}\right|^{p-1} u_{\mu_{j}}}{p}\right) d x-\left(1-\frac{1}{p}\right)\left|\left\{u_{\mu_{j}} \leq-1\right\}\right|\right]=c_{\mu_{j}} \geq c_{0}
\end{aligned}
$$

by (3.1) and

$$
\begin{aligned}
I_{\mu_{j}}^{\prime}\left(u_{\mu_{j}}\right) v=\int_{\Omega}\left(\left|\nabla u_{\mu_{j}}\right|^{p-2} \nabla u_{\mu_{j}} \cdot \nabla v-\lambda u_{\mu_{j}+}^{p-1} v-u_{\mu_{j^{+}}}^{p^{*}-1} v\right) d x+\mu_{j} & {\left[\int_{\left\{u_{\mu_{j}} \geq 0\right\}} v d x\right.} \\
& \left.+\int_{\left\{-1<u_{\mu_{j}}<0\right\}}\left(1-\left|u_{\mu_{j}}\right|^{p-1}\right) v d x\right]=0 \quad \forall v \in W_{0}^{1, p}(\Omega),
\end{aligned}
$$

and passing to the limits gives

$$
\int_{\Omega}\left(\frac{|\nabla u|^{p}}{p}-\frac{\lambda u_{+}^{p}}{p}-\frac{u_{+}^{p^{*}}}{p^{\star}}\right) d x \geq c_{0}
$$

and

$$
\int_{\Omega}\left(|\nabla u|^{p-2} \nabla u \cdot \nabla v-\lambda u_{+}^{p-1} v-u_{+}^{p^{*}-1} v\right) d x=0 \quad \forall v \in W_{0}^{1, p}(\Omega)
$$

so $u$ is a nontrivial weak solution of the problem

$$
\left\{\begin{aligned}
-\Delta_{p} u & =\lambda u_{+}^{p-1}+u_{+}^{p^{*}-1} & & \text { in } \Omega \\
u & =0 & & \text { on } \partial \Omega .
\end{aligned}\right.
$$

Then $u>0$ in $\Omega$ and its interior normal derivative $\partial u / \partial v>0$ on $\partial \Omega$ by the strong maximum principle and the Hopf lemma for the $p$-Laplacian (see Vázquez [17]). Since $u_{\mu_{j}} \rightarrow u$ in $C_{0}^{1}(\bar{\Omega})$, then $u_{\mu_{j}}>0$ in $\Omega$ for all sufficiently large $j$.

It remains to show that $u_{\mu}$ minimizes $I_{\mu}$ on $\mathcal{N}_{\mu}$ when it is positive. For each $w \in \mathcal{N}_{\mu}$, we will construct a path $y_{w} \in \Gamma$ such that

$$
\max _{u \in y_{w}([0,1])} I_{\mu}(u)=I_{\mu}(w) .
$$

Since

$$
I_{\mu}\left(u_{\mu}\right)=c_{\mu} \leq \max _{u \in y_{w}([0,1])} I_{\mu}(u)
$$

by the definition of $c_{\mu}$, the desired conclusion will then follow. First we note that the function

$$
g(t)=I_{\mu}(t w)=\frac{t^{p}}{p} \int_{\Omega}\left(|\nabla w|^{p}-\lambda w^{p}\right) d x-\frac{t^{p^{*}}}{p^{\star}} \int_{\Omega} w^{p^{*}} d x+\mu t \int_{\Omega} w d x, \quad t \geq 0
$$

has a unique maximum at $t=1$. Indeed,

$$
\begin{aligned}
g^{\prime}(t)=t^{p-1} \int_{\Omega}\left(|\nabla w|^{p}-\lambda w^{p}\right) d x-t^{p^{*}-1} \int_{\Omega} w^{p^{*}} d x+\mu \int_{\Omega} w d x & \\
& =\left(t^{p-1}-t^{p^{*}-1}\right) \int_{\Omega}\left(|\nabla w|^{p}-\lambda w^{p}\right) d x+\left(1-t^{p^{*}-1}\right) \mu \int_{\Omega} w d x
\end{aligned}
$$


since $w \in \mathcal{N}_{\mu}$, and the last two integrals are positive since $\lambda<\lambda_{1}$ and $w>0$, so $g^{\prime}(t)>0$ for $0 \leq t<1$, $g^{\prime}(1)=0$, and $g^{\prime}(t)<0$ for $t>1$. Hence

$$
\max _{t \geq 0} I_{\mu}(t w)=I_{\mu}(w)>0
$$

since $g(0)=0$. In view of Lemma 3.1 (ii), now it suffices to observe that there exists $\widetilde{R}>\max \{1, R\}$ such that

$$
I_{\mu}(\widetilde{R} u)=\frac{\widetilde{R}^{p}}{p} \int_{\Omega}\left(|\nabla u|^{p}-\lambda u^{p}\right) d x-\frac{\widetilde{R}^{p^{*}}}{p^{\star}} \int_{\Omega} u^{p^{*}} d x+\mu \widetilde{R} \int_{\Omega} u d x \leq 0
$$

for all $u$ on the line segment joining $w$ to $v_{\varepsilon}$ since all norms on a finite dimensional space are equivalent.

Acknowledgement: The third author was supported by the National Research Foundation of Korea Grant funded by the Korea Government (MEST) (NRF-2015R1D1A3A01019789).

\section{References}

[1] Alfonso Castro, Djairo G. de Figueredo, and Emer Lopera. Existence of positive solutions for a semipositone $p$-Laplacian problem. Proc. Roy. Soc. Edinburgh Sect. A, 146(3):475-482, 2016.

[2] M. Chhetri, P. Drábek, and R. Shivaji. Existence of positive solutions for a class of $p$-Laplacian superlinear semipositone problems. Proc. Roy. Soc. Edinburgh Sect. A, 145(5):925-936, 2015.

[3] S. Unsurangie. Existence of a solution for a wave and elliptic Dirichlet problem. PhD thesis, University of North Texas, Denton, 1988.

[4] W. Allegretto, P. Nistri, and P. Zecca. Positive solutions of elliptic nonpositone problems. Differential Integral Equations, 5(1):95-101, 1992.

[5] A. Ambrosetti, D. Arcoya, and B. Buffoni. Positive solutions for some semi-positone problems via bifurcation theory. Differential Integral Equations, 7(3-4):655-663, 1994.

[6] Scott Caldwell, Alfonso Castro, Ratnasingham Shivaji, and Sumalee Unsurangsie. Positive solutions for classes of multiparameter elliptic semipositone problems. Electron. J. Differential Equations, pages No. 96, 10 pp. (electronic), 2007.

[7] Mohammed Guedda and Laurent Véron. Quasilinear elliptic equations involving critical Sobolev exponents. Nonlinear Anal., 13(8):879-902, 1989.

[8] Haïm Brézis and Louis Nirenberg. Positive solutions of nonlinear elliptic equations involving critical Sobolev exponents. Comm. Pure Appl. Math., 36(4):437-477, 1983.

[9] P.-L. Lions. On the existence of positive solutions of semilinear elliptic equations. SIAM Rev., 24(4):441-467, 1982.

[10] Djairo G. de Figueiredo, Jean-Pierre Gossez, and Pedro Ubilla. Local "superlinearity" and "sublinearity" for the $p$ Laplacian. J. Funct. Anal., 257(3):721-752, 2009.

[11] Gerald B. Folland. Real analysis. Pure and Applied Mathematics (New York). John Wiley \& Sons, Inc., New York, second edition, 1999. Modern techniques and their applications, A Wiley-Interscience Publication.

[12] P.-L. Lions. The concentration-compactness principle in the calculus of variations. The limit case. I. Rev. Mat. Iberoamericana, 1(1):145-201, 1985.

[13] P.-L. Lions. The concentration-compactness principle in the calculus of variations. The limit case. II. Rev. Mat. Iberoamericana, 1(2):45-121, 1985.

[14] Kanishka Perera, Ravi P. Agarwal, and Donal O'Regan. Morse theoretic aspects of p-Laplacian type operators, volume 161 of Mathematical Surveys and Monographs. American Mathematical Society, Providence, RI, 2010.

[15] Pavel Drábek and Yin Xi Huang. Multiplicity of positive solutions for some quasilinear elliptic equation in $\mathbf{R}^{N}$ with critical Sobolev exponent. J. Differential Equations, 140(1):106-132, 1997.

[16] Antonio Ambrosetti and Paul H. Rabinowitz. Dual variational methods in critical point theory and applications. J. Functional Analysis, 14:349-381, 1973.

[17] J. L. Vázquez. A strong maximum principle for some quasilinear elliptic equations. Appl. Math. Optim., 12(3):191-202, 1984. 